\title{
Singular Supports of Solutions of Partial Differential Equations in a Slab Domain
}

\author{
By
}

\author{
Kinimasa NisHIWADA*
}

\section{$\S 1$. Introduction}

In [6], F. John proved that for a differential operator, non-solvability to the non-characteristic Canchy problem for any initial data with compact support is equivalent to rather stringent non-hyperbolicity. ${ }^{1)}$ In the present paper, we shall study an analogous question where we shall be interested not in the support but in the singular support of solution. To state more precisely, let $D$ denote the imaginary gradient $-i\left(\frac{\partial}{\partial x_{1}}, \cdots, \frac{\partial}{\partial x_{n}}\right)$ and $P(D)$ be a differential operator with constant coefficients obtained from a polynomial $P(\xi)$ of $n$ variables $\xi=\left(\xi_{1}, \ldots, \xi_{n}\right)$. Our main result is the following

Theorem 1. Assume that the polynomial $P(\xi)$ has the form

$$
P(\xi)=a \xi_{1}^{l}+\sum_{\alpha_{1}<l} a_{\alpha} \xi^{\alpha}
$$

with $a \neq 0$, and that the zeros of the polynomial have the property;

$$
\operatorname{Im} \zeta_{1} \rightarrow \infty \text { when } \xi^{\prime} \rightarrow \infty \text { in } \boldsymbol{R}^{n-1} \text { and } P\left(\zeta_{1}, \xi^{\prime}\right)=0 \text {. }
$$

Under these assumptions, if the equation

$$
P(D) u=f
$$

in a slab domain $\Omega=\left\{x \in \boldsymbol{R}^{n} ; c_{1}<x_{1}<c_{2}\right\}$, admits a solution $u \in \mathscr{D}^{\prime}(\Omega)$ with bounded singular support for a given $f \in C^{\infty}(\Omega)$, then we have that

Communicated by S. Matsuura, February 26, 1974.

* Graduate School, Kyoto University, Kyoto.

1) Cf. Matsuura [8] for the extension to general systems. 
$u \in C^{\infty}(\Omega)$.

The proof of Theorem 1 will be given in section 2 . In section 3 , we shall state and prove an analogous theorem for Gevrey classes. In section 4. we shall consider partial converses of these theorems by constructing suitable fundamental solutions, inspired by an idea due to Kashiwara [7].

The author wishes to express his gratitude to Professor Shigetake Matsuura for suggesting the present problem and for his helps.

\section{§. Proof of Theorem 1}

We shall first consider to what extent the regularity of solutions $u$ can be deduced from the condition (1.1).

Lemma 1. A polynomial $P(\xi)$ can be written in the form (1.1) if and only if there exist positive constants $\varepsilon, \rho, C$ with $0<\rho \leqq 1$ such that

$$
\left|P^{(\alpha)}(\xi) P(\xi)\right| \leqq C(1+|\xi|)^{-\alpha_{1}-\rho \mid \alpha^{\prime}}
$$

for all multi-indices $\alpha=\left(\alpha_{1}, \alpha^{\prime}\right)$ and $\xi \in \Gamma$, where $\Gamma=\left\{\xi \in R^{n} ;\left|\xi^{\prime}\right|<\varepsilon\left|\xi_{1}\right|^{\rho}\right\}$.

Proof. Suppose that $P$ has the form $P(\xi)=a \xi_{1}^{l}\left(1+\sum_{\alpha 1<l} a_{\alpha} \xi_{1}^{\alpha_{1}-l} \xi^{\prime \alpha^{\prime}}\right)$ for $\xi_{1} \neq 0$. If $\rho=\inf \left(\left(l-\alpha_{1}\right) /\left|\alpha^{\prime}\right|, 1\right)$, the infimum being taken over all $\alpha$ with $a_{\alpha} \neq 0$, and if $\varepsilon>0$ is sufficiently small, then we have

$$
\begin{aligned}
& \frac{1}{2}\left|a \xi_{1}^{l}\right| \leqq|P(\xi)| \leqq \frac{3}{2}\left|a \xi_{1}^{l}\right|, \xi \in \Gamma \\
& \left|P^{(\beta)}(\xi)\right| \leqq C\left|\xi_{1}\right|^{l-\beta_{1}-\rho\left|\beta^{\prime}\right|}, \xi \in \Gamma
\end{aligned}
$$

which proves the estimate (2.1). Conversely if the highest order tern of $P(\xi)$ with respect to $\xi_{1}$ is dependent on the other variables, there exists a multi-index $a \neq 0$ such that $P^{(\alpha)}(\xi)$ has the same order with respect to $\xi_{1}$ as $P(\xi)$ and such that the term is independent of the other variables. Thus $\left|P^{(\alpha)}(\xi) / P(\xi)\right|$ does not converge to zero when $\xi_{1} \rightarrow \infty$ and $\xi^{\prime}$ bounded, which proves the lemma.

Lemma 2. Assume that there is a subset $\Gamma \subset \boldsymbol{R}^{n}$ and a constant 
$\rho>0$ such that

$$
\left|P^{(\alpha)}(\xi) / P(\xi)\right| \leqq C(1+|\xi|)^{-\rho|\alpha|}, \xi \in \Gamma .
$$

If $P(D) u=f \in C^{\infty}(U)$ where $U$ is an open subset in $\mathbb{R}^{n}$, it follows that for every $\varphi \in C_{0}^{\infty}(U)$ there exist constants $C_{N}$, such that

$$
|\widehat{\varphi} \hat{u}(\xi)| \leqq C_{N}(1+|\xi|)^{-N}, N=1,2, \cdots, \xi \in \Gamma .
$$

Proof. Let us consider a solution $v(x, \xi)$ of the equation

$$
{ }^{t} P(D) v=\varphi e^{-i<x, \xi>}
$$

where ${ }^{t} P(D)=P(-D)$ is the formal adjoint of $P$. If we set $v(x, \xi)=$ $e^{-i<x, \xi>} w(x, \xi) / P(\xi)$ when $\xi \in \Gamma$, Leibniz formula gives

$$
{ }^{t} P(D) v(x, \xi)=\left(w(x, \xi)+\sum_{\alpha \neq 0} \frac{P^{(\alpha)}(\xi)}{P(\xi)} \frac{D^{\alpha}}{\alpha !} w(x, \xi)\right) \mathrm{e}^{-i<x, \xi>} .
$$

Hence (2.3) is equivalent to the equation

$$
w-R(\xi, D) w=\varphi, \xi \in \Gamma,
$$

where $R(\xi, D)=-\sum_{\alpha \neq 0}\left(P^{\alpha}(\xi) D^{\alpha} / \alpha ! P(\xi)\right)$. Suggested by the formal solution $w=\sum_{0}^{\infty} R(\xi, D)^{k} \varphi$ to (2.4), we consider the following approximate solutions

$$
\begin{aligned}
& w_{N}=\sum_{0}^{N} R(\xi, D)^{k} \varphi, \\
& v_{N}=e^{-i<x, \xi>} w_{N} ! P(\xi) .
\end{aligned}
$$

The same calculation gives

$$
\begin{aligned}
{ }^{t} P(D) v_{N}(x, \xi) & =\left(w_{N}-R(\xi, D) w_{N}\right) e^{-i<x, \xi>} \\
& =\left(\varphi(x)-R(\xi, D)^{N+1} \varphi(x)\right) e^{-i<x, \xi>} .
\end{aligned}
$$

Then it follows that

$$
\begin{aligned}
\widehat{\varphi}(\xi) & =\int u(x) \cdot \varphi(x) e^{-i<x, \xi>} d x \\
& =\int u(x)\left(R(\xi, D)^{N+1} \varphi\right) e^{-i<x, \xi>} d x \\
& +\int f(x) \frac{w_{N}(x, \xi)}{P(\xi)} e^{-i<x, \xi>} d x, \xi \in \Gamma .
\end{aligned}
$$


Since the order of distributions $\left(D^{\alpha} \varphi\right) u$ is independent of $\alpha$, using (2.2) we can estimate the first term on the right-hand side of (2.5) by

$$
C_{N}(1+|\xi|)^{-\rho(N+1)+M}, N=1,2, \ldots, \quad \xi \in \Gamma
$$

with some constants $C_{N}$ and $M$. The second term also decreases rapidly in $\Gamma$ for $w_{N} f \in C_{0}^{\infty}$ and every derivative $D_{x}^{\alpha}\left(w_{N}(x, \xi) / P(\xi)\right)$ is bounded when $\xi \in \Gamma$. The proof is now complete.

Proof of Theorem 1. Since the preceding lemmas show that for each $\varphi \in C_{0}^{\infty}(\Omega) \varphi u(\xi)$ is rapidly decreasing when $\left|\xi^{\prime}\right|<\varepsilon\left|\xi_{1}\right|^{\rho}$, our aim now is to prove the same fact also when $\left|\xi^{\prime}\right| \geqq \varepsilon\left|\xi_{1}\right| \rho$. In doing so we assume in what follows without loss of generality that $\Omega=\left\{x ;-2<x_{1}<2\right\}, a=$ 1 in (1.1) and that bounded is supp $u$ as well as sing supp $u$. Moreover we can assume that $u \in C^{0}$ when $-1 \leqq x_{1} \leqq 1$. In fact, if we take a test function $\phi\left(x_{1}\right) \in C_{0}^{\infty}((-2,2))$ which is equal to 1 in a neighborhood of the interval $\left[-\frac{3}{2}, \frac{3}{2}\right]$ and if we replace $p(D)$ by $P(D)(1-\Delta)^{k}$ and $u$ by $(1-$ $\Delta)^{-k} \phi u$ in the statement of the theorem, then to prove the theorem is equivalent to do it in this case at least when $-\frac{3}{2}<x_{1}<\frac{3}{2}$, and the fact that $(1-\Delta)^{-k} \phi u \in C^{0}$ when $k$ is large assures the claim. The partial Fourier transforms of $u$ and $f$,

$$
\begin{array}{ll}
\hat{u}_{1}\left(x_{1}, \xi^{\prime}\right)=\int u(x) e^{-i<x^{\prime}, \xi^{\prime}>} d x^{\prime}, & -1 \leqq x_{1} \leqq 1, \\
\hat{f}_{1}\left(x_{1}, \xi^{\prime}\right)=\int f(x) e^{-i<x^{\prime}, \xi^{\prime}>} d x^{\prime}, & -1 \leqq x_{1} \leqq 1,
\end{array}
$$

are then analytic functions of $\xi^{\prime}$ for fixed $x_{1}$. Since $P(D) u=f$, it follows that

$$
P\left(D_{1}, \xi^{\prime}\right) \hat{u}_{1}\left(x_{1}, \xi^{\prime}\right)=\hat{f}_{1}\left(x_{1}, \xi^{\prime}\right), \quad-1 \leqq x_{1} \leqq 1
$$

We shall now prove that there exist constants $C, C_{N}$ and $M$ which are independent of $x_{1},-1 \leqq x_{1} \leqq 1$, such that

$$
\begin{aligned}
& \left|D_{1}^{k} \hat{u}_{1}\left(x_{1}, \xi^{\prime}\right)\right| \leqq C\left(1+\left|\xi^{\prime}\right|\right)^{M}, \quad 1 \leqq k \leqq l,-1 \leqq x_{1} \leqq 1 \\
& \left|\hat{f}_{1}\left(x_{1}, \xi^{\prime}\right)\right| \leqq C_{N}\left(1+\left|\xi^{\prime}\right|\right)^{-N}, \quad N=1,2, \ldots .
\end{aligned}
$$


To do so we choose a function $\phi \in C_{0}^{\infty}((-2,2))$ such that $\phi=1$ when $-1 \leqq x_{1} \leqq 1$ and set $v=\phi u$. Thus we have when $-1 \leqq x_{1} \leqq 1$

$$
\begin{aligned}
& D_{1}^{k} \hat{u}_{1}\left(x_{1}, \xi^{\prime}\right)=D_{1}^{k} \hat{v}_{1}\left(x_{1}, \xi^{\prime}\right) \\
& =(2 \pi)^{-1} \int_{-\infty}^{\infty} \xi_{1}^{k} \hat{v}(\xi) e^{i x_{1} \xi_{1}} d \xi_{1} .
\end{aligned}
$$

Since $v \in \mathcal{E}^{\prime}(\Omega)$, we obtain for some constants

$$
|\hat{v}(\xi)| \leqq C(1+|\xi|)^{M}
$$

Moreover Lemma 1 and 2 give that when $\left|\xi^{\prime}\right|<\varepsilon\left|\xi_{1}\right|^{\rho}$

$$
|\hat{v}(\xi)| \leqq C_{N}(1+|\xi|)^{-N}, \quad N=1,2, \ldots
$$

Applying these estimates to (2.9) we obtain

$$
\begin{aligned}
& \left|D_{1}^{k} \hat{u}_{1}\left(x_{1}, \xi^{\prime}\right)\right| \leqq !(2 \pi)^{-1} C \int_{\left|\xi_{1}\right| \leqq \varepsilon^{-1 / \rho_{\left|\xi^{\prime}\right|}}} \xi_{1}^{k}(1+|\xi|)^{M} d \xi_{1} \mid \\
& \quad+\left|(2 \pi)^{-1} C_{N} \int_{\left|\xi_{1}\right| \geq \varepsilon^{-1 / \rho_{\mid \xi^{\prime}} \mid}} \xi_{1}^{k}(1+|\xi|)^{-N} d \xi_{1}\right|
\end{aligned}
$$

Since the second integral in (2.10) is absolutely convergent when $N>l+1$, (2.7) follows with other constants $C$ and $M$. (2.8) also follows similary.

Now decomposing the ordinary differential equation (2.6), we obtain

$$
\left(D_{1}-\sigma_{1}\left(\xi^{\prime}\right)\right) \ldots\left(D_{1}-\sigma_{l}\left(\xi^{\prime}\right)\right) \hat{u}_{1}\left(x_{1}, \xi^{\prime}\right)=\hat{f}_{1}\left(x_{1}, \xi^{\prime}\right)
$$

where $\sigma_{1}\left(\xi^{\prime}\right), \ldots, \sigma_{l}\left(\xi^{\prime}\right)$ are the roots of the polynomial $P\left(\xi_{1}, \xi^{\prime}\right)$ of $\xi_{1}$, which satisfy the following estimates with positive constants $C_{1}, C_{2}, \delta_{1}$ and $\delta_{2}$

$$
C_{1}\left|\xi^{\prime}\right|^{\delta_{1}} \leqq\left.\operatorname{Im} \sigma_{k}\left(\xi^{\prime}\right)|\leqq| \sigma_{k}\left(\xi^{\prime}\right)\left|\leqq C_{2}\right| \xi^{\prime}\right|^{\delta_{2}}, \quad k=1,2, \ldots l
$$

when $\xi^{\prime}$ is large. For the first inequality follows from the hypothesis (1.2) (see Lemma 2.1 in Appendix in Hörmander [4]) and the last from the usual estimate of the roots of a polynomial by its coefficients. Now we introduce the functions $w_{k}\left(x_{1}, \xi^{\prime}\right), 0 \leqq k \leqq l$, by the equations

$$
\left(D_{1}-\sigma_{k+1}\left(\xi^{\prime}\right)\right) w_{k+1}\left(x_{1}, \xi^{\prime}\right)=w_{k}\left(x_{1}, \xi^{\prime}\right), \quad 0 \leqq \mathrm{k} \leqq l-1,
$$

where $w_{0}\left(x_{1}, \xi^{\prime}\right)=\hat{f}_{1}\left(x_{1}, \xi^{\prime}\right)$ and $w_{l}\left(x_{1}, \xi^{\prime}\right)=\hat{u}_{1}\left(x_{1}, \xi^{\prime}\right)$. In view of (2.7) and (2.11) it follows that there are constants $C$ and $M$ such that 


$$
\left|w_{k}\left(x_{1}, \xi^{\prime}\right)\right| \leqq C(1+|\xi|)^{M}, \quad-1 \leqq x_{1} \leqq 1
$$

Solving the equation when $k=0$ in (2.2), we have

$$
w_{1}\left(x_{1}, \xi^{\prime}\right)=e^{-i \sigma_{1}\left(t-x_{1}\right)} w_{1}\left(t, \xi^{\prime}\right)-i \int_{x_{1}}^{t} e^{-i \sigma_{1}\left(s-x_{1}\right)} \hat{f_{1}}\left(s, \xi^{\prime}\right) d s
$$

where $-1 \leqq x_{1}, t \leqq 1$. If we restrict the range of $x_{1}$ to the interval $\left[-\frac{1}{2}, \frac{1}{2}\right]$, we can always choose $t$ so that $\operatorname{Im} \sigma_{1}\left(\xi^{\prime}\right)\left(t-x_{1}\right)=-\frac{1}{2}$ $\left|\operatorname{Im} \sigma_{1}\left(\xi^{\prime}\right)\right|$. Thus we have

$$
\left|w_{1}\left(x_{1}, \xi^{\prime}\right)\right| \leqq\left|w_{1}\left(x_{1} \pm \frac{1}{2}, \xi^{\prime}\right)\right| e^{-\mid m_{\sigma_{1}} / 2}+\sup _{-1 \leqq x_{1} \leqq 1}\left|\hat{f}\left(x_{1}, \xi^{\prime}\right)\right|
$$

In view of (2.8), (2.11) and (2.13), this means that there exist constants $C_{N}$ such that

$$
\left|w_{1}\left(x_{1}, \xi^{\prime}\right)\right| \leqq C_{N}\left(1+\left|\xi^{\prime}\right|\right)^{-N} \quad N=1,2, \ldots, \quad-\frac{1}{2} \leqq x_{1} \leqq \frac{1}{2} .
$$

Repeating the same arguments to the rest equations in (2.12), we obtain finally

$$
\left|\hat{u}_{1}\left(x_{1}, \xi^{\prime}\right)\right| \leqq C_{N}\left(1+\left|\xi^{\prime}\right|\right)^{-N}, \quad N=1,2, \ldots,
$$

when $x_{1}$ is in a neiborhood of 0 . Since this condition, however, plays no essential role, we may assume that $(2.15)$ is valid also when $-1 \leqq x_{1} \leqq$ 1. Since in the region defined by the inequality, $\left|\xi^{\prime}\right| \geqq \varepsilon\left|\xi_{1}\right| \rho$,

$$
1+|\xi| \leqq C\left(1+\left|\xi^{\prime}\right|\right)^{1 / \rho}
$$

with a constant $C$, for each $\varphi \in C_{0}^{\infty}\left(R^{\prime}\right)$ such that supp $\varphi \subset[-1,1]$ we have according to (2.15)

$$
\begin{aligned}
|\widehat{\varphi}(\xi)| & =\left|\int_{-\infty}^{\infty} \varphi\left(x_{1}\right) \hat{u}_{1}\left(x_{1}, \xi^{\prime}\right) e^{-i x_{1} \xi_{1}} d x_{1}\right| \\
& \leqq 2 C_{N} \sup |\varphi| \cdot C^{\rho N}(1+|\xi|)^{-\rho N}, \quad N=1,2, \ldots,
\end{aligned}
$$

when $\left|\xi^{\prime}\right| \geqq \varepsilon\left|\xi_{1}\right| \rho$. Adding the result obtained from the lemmas we conclude that $u \in C^{\infty}$ under the condition, $-1<x_{1}<1$, which we can of course remove. The proof of the theorem is complete. 
Examples. The hypoelliptic operators satisfy both the conditions (1.1) and (1.2), although the theorem in this case is a consequence of the well known regularity theorem. On the other hand, the polynomial $P\left(\xi_{1}, \xi_{2}, \xi_{3}\right)=\xi_{1}^{2}+\xi_{2}^{2}+\xi_{2}^{2} \xi_{3}^{2}+\xi_{3}^{2}$, clearly satisfying the conditions, is not hypoelliptic. In fact the highest order term with respect to $\xi_{2}$ is dependent on $\xi_{3}$.

\section{$\S 3$. Gevrey case}

We shall consider here the case when in Theorem $1 f$ will belong to a Gevrey class which will be introduced as follows.

Definition 1. Let $\Omega$ be an open set in $\mathbb{R}^{n}$. We denote by $\Gamma^{\rho}(\Omega)$, $0<\rho \leqq 1$, the set of functions $f$ in $C^{\infty}(\Omega)$ such that for every compact set $K \subset \Omega$ there is a constant $C$ for which the inequality

$$
\left|D^{\alpha} f(x)\right| \leqq C(C|\alpha|)^{|\alpha| / \rho}, x \in K
$$

is valid for every multi-index $\alpha$. We slao set $\Gamma_{0}^{\rho}=\Gamma^{\rho} \cap C_{0}^{\infty}$ and define the notation $\Gamma^{\rho}$ sing supp $u$ for a distribution $u$ in $\Omega$ as the smallest subset outside whicn $u \in \Gamma^{\rho}$.

The definition above is a special case of Definition 4.4.2. in Hörmander [4]. The following lemma is an easy consequence of (3.1).

Lemma 3. When $\rho<1, u \in \mathscr{D}^{\prime}(\Omega)$ belongs to $\Gamma^{\rho}(\Omega)$ if and only if for each $\varphi \in \Gamma_{0}^{\rho}$ there exists a constant $C$ which is independent of $N$ such that

$$
\widehat{\mid \varphi \mathcal{u}}(\xi) \mid \leqq C(C N)^{N}(1+|\xi|)^{-\rho N}, \quad N=1,2, \ldots, \quad \xi \in \mathbb{R}^{n}
$$

or equivalently that

$$
\widehat{\varphi} \boldsymbol{u}(\xi) \mid \leqq C(C N)^{N / \rho}(1+|\xi|)^{-N}, \quad N=1,2, \ldots, \quad \xi \in \mathbb{R}^{n}
$$

which follows from (3.2) replacing $N$ by $N / \rho$.

Proof. If $u \in \Gamma^{\rho}(\Omega)$, we have for each $\varphi \in \Gamma_{\mathbf{0}}^{\rho}(\Omega)$ and each multiindex $a$ 


$$
\left|\xi^{\alpha} \widehat{\varphi} u(\xi)\right|=\int_{\operatorname{supp} \varphi} e^{-i<x, \xi>} D^{\alpha}(\varphi u) d x
$$

which means from (3.1) when $|a| \leqq N$ that

$$
|\widehat{\varphi} u(\xi)| \leqq C(C N)^{N / \rho}(1+|\xi|)^{-N}
$$

Conversely let $u \in \mathscr{D}^{\prime}(\Omega)$ satisfy the condition (3.2). If we note that for every compact set $K \subset \Omega$ we can shoose a function $\varphi \in \Gamma_{0}^{\rho}(\Omega)$ such that $\varphi=1$ near $K$ (see e.g. Lemma 5.7.1. in [4]), we have for such $\varphi$

$$
\begin{aligned}
D^{\alpha} u(x) & =D^{\alpha}(\varphi u)(x) \\
& =(2 \pi)^{-n} \int e^{i<x, \xi>\xi^{\alpha}} \hat{\varphi} u(\xi) d \xi, \quad x \in K .
\end{aligned}
$$

If we choose $N=n+1+|\alpha|$ in (3.3), we obtain the estimate,

$$
\left|D^{\alpha} u(x)\right| \leqq C(C(|\alpha|+n+1))^{(|\alpha|+n+1)<\rho}, \quad x \in K
$$

If we note that when $(N+K)^{k / N}<2$ and $N \geqq K$

$$
(N+k)^{N+k} \leqq(4 N)^{N}
$$

(3.4) implies that (3.1) is valid with another constant when $x \in K$, which complete the proof.

Now we shall have instead of Theorem 1 the following

Theorem 2. Let the notations and the hypotheses be as in Theorem 1. In addition suppose the following conditions;

(i) $\left|\operatorname{Im} \xi_{1}\right| \geqq C\left|\xi^{\prime}\right|^{\delta_{1}}, 0<C$, when $P\left(\xi_{1}, \xi^{\prime}\right)=0$ and real $\xi^{\prime}$ is large,

(ii) $\Gamma^{\delta_{2}}$ sing supp $u$ is bounded in $\Omega$.

(iii) $f \in \Gamma^{\delta_{3}}(\Omega)$,

then if $0<\delta=\min \left(\delta_{1}, \delta_{2}, \delta_{3}\right)<1$ it follows that $u \in \Gamma^{\rho \delta}(\Omega)$ where $\rho$ is the number introduced in Lemma 1.

The verification of the present theorem is a routine repetition of that of Theorem 1 except for some estimates concerning with Lemma 3 . At first Lemma 2 will be replaced by the following

Lemma 4. Besides the hypotheses in Lemma 2 if $f \in \Gamma^{\rho_{0}}(U)$ it follows that for each $\varphi \in \Gamma_{0}^{\rho_{1}}, \rho_{1}=\min \left(\rho, \rho_{0}\right)$, 


$$
|\widehat{\varphi}(\xi)| \leqq C(C N)^{N}(1+|\xi|)^{-\rho_{1} N}, \quad N=1,2, \ldots, \quad \xi \in \Gamma .
$$

Proof. Although the proof will be carried on parallel to that of Lemma 2 , we must define $w_{N}$ more carefully, that is, we set

$$
\begin{aligned}
& w_{N}=\sum_{\left|\alpha_{1}\right|+\ldots+\mid \alpha_{k} \leq N-m}(-1)^{k}\left(P^{\left(\alpha_{1}\right)}(\xi) \ldots P^{\left(\alpha_{k}\right)}(\xi) D^{\left.\alpha_{1}+\cdots+\alpha_{k} / \alpha_{1} ! \ldots \alpha_{k} ! P(\xi)^{k}\right) \varphi}\right. \\
& \quad k=1,2, \ldots,
\end{aligned}
$$

where $m=\operatorname{deg} P$. Thus we have

$$
\begin{aligned}
& w_{N}-R(\xi, D) w_{N} \\
& =\varphi-\sum_{N-m \leqq\left|\alpha_{1}+\ldots+\right| \alpha_{k} \leqq N}^{\prime}(-1)^{k}\left(P^{\left(\alpha_{1}\right)}(\xi) \ldots P^{\left(\alpha_{k}\right)}(\xi) D^{\left.\alpha_{1}+\cdots+\alpha_{k} / \alpha_{1} ! \ldots \alpha_{k} ! P(\xi)^{k}\right) \varphi}\right.
\end{aligned}
$$

where $\Sigma^{\prime}$ means a partial summation in the range noted below. Since each term in the summation $\Sigma^{\prime}$ is bounded by $C(C N)^{N}(1+|\xi|)^{-\rho(N-m)}$ and the number of the terms in $\Sigma^{\prime}$ does not exceed $A^{N}$ with a constant $A$ independent of $N$, the summation $\Sigma^{\prime}$ can be estimated when $\xi \in \Gamma$ by $C(C N)^{N}(1+|\xi|)^{-\rho(N-m)}, N=1,2, \ldots$, with $C$ independent of $N$. If we note that the coefficient of each term in $w_{N} / P(\xi)$ is bounded when $\xi \in \Gamma$ and that the number of its terms does not exceed $A^{N}$, using (2.5) and (3.5) we obtain (3.6).

Proof of Theorem 2. We may of course assume that $\delta=\delta_{1}=\delta_{2}=\delta_{3}$. We shall first replace $(2.8)$ by

$$
\left|\hat{f}_{1}\left(x_{1}, \xi^{\prime}\right)\right| \leqq C(C N)^{N}\left(1+\left|\xi^{\prime}\right|\right)^{-\delta N}, \quad N=1,2, \ldots,
$$

following the hypothesis that $f \in \Gamma^{\delta}(\Omega)$. When we utilize the estimate (2.14) we note the elementary fact such that for any positive constants $\varepsilon, M$ there is a constant $C$ independent of $N$ such that

$$
(1+\tau)^{M} \exp \left(-\varepsilon \tau^{\delta}\right) \leqq C(C N)^{N}(1+\tau)^{-\delta N}, \quad \tau>0, \quad N=1,2, \ldots,
$$

obtained by calculating the value $\tau_{0}$ which makes the left-hand side of (3.9) maximum. Then we have instead of (2.15)

$$
\left|\hat{u}_{1}\left(x_{1}, \xi^{\prime}\right)\right| \leqq C(C N)^{N}\left(1+\left|\xi^{\prime}\right|\right)^{-\delta N}, \quad N=1,2, \ldots
$$

Thus in view of (2.16) we have proved Theorem 2. 


\section{$\S 4$. Fundamental Solutions with Singular Supports in a Proper Cone}

In this section we shall show roughly speaking that if contrary to the condition (1.2) of Theorem 1 imaginary parts of some roots are bounded when $\xi^{\prime} \in \boldsymbol{R}^{n-1}$ while those of the other roots go to infinity, we can construct a solution which has actually a bounded singular support in $\Omega$.

Theorem 3. When $0<\rho<1$, there exists a fundamental solution $E \in D^{\prime}(\Omega)$ of $P(D)$ such that $\Gamma^{\rho}$ sing supp $E$ is contained in a $\vartheta$-proper cone, that is, in a cone contained in $\{x ;<x, \vartheta>>0\} \cup\{0\}$ if and only if for some positive constants $\varepsilon, \tau_{0}, t_{0}$ and a neighborhood $U$ of $\vartheta$

$$
P(\xi+i t \eta) \neq 0, \quad-\varepsilon|\xi|^{\rho}<t<-t_{0}, \quad|\xi| \geqq \tau_{0},
$$

when $\eta \in U$.

Proof. Assume that such a solution $E$ exists. Choosing a function $\psi \in \Gamma_{0}^{\rho}$ such that $\psi=1$ near the origin, we have

$$
P(D) f(x)=\delta(x)+g(x)
$$

where $f=\psi E$ and $g=P(D)((\psi-1) E)$. Since $f, g \in \mathcal{E}^{\prime}\left(\boldsymbol{R}^{n}\right)$, taking the Fourier-Laplace transforms of (4.2), we have

$$
P(\zeta) \hat{f}(\zeta)=1+g(\zeta), \quad \zeta \in C^{n} .
$$

we now note that $\Gamma^{\rho}$ sing supp $g \subset \Gamma^{\rho}$ sing supp $E$ and does not intersect a neighborhood of the origin, which makes it possible to choose a compact neighborhood $K$ of $\Gamma^{\rho}$ sing supp $g$ and a neighborhood $U$ of $\vartheta$ such that $h(-\eta)<$ const. $<0$ when $\eta \in U$, where $h(\xi)$ is the support function of $K ; h(\xi)=\sup _{x \in K}\langle x, \xi\rangle$.

Writing that $g=g_{1}+g_{2}$ where $g_{1} \in \mathcal{E}^{\prime}$, supp $g_{1} \subset K$ and $g_{2} \in \Gamma_{0}^{\rho}$, we have in view of the Paley-Wiener theorem and the proof of Lemma 5.7.2. in Hörmander [4]

$$
\begin{aligned}
& \left|\hat{g}_{1}(\zeta)\right| \leqq C(1+|\zeta|)^{M} e^{h(\operatorname{Im} \zeta)} \\
& \left|\hat{g}_{2}(\zeta)\right| \leqq C \exp \left(A|\operatorname{Im} \zeta|-B\left|R_{e} \zeta\right|^{\rho}\right)
\end{aligned}
$$


for some positive constants $C, A, B$ and $M$. Since (4.3) implies that when $P(\zeta)=0,\left|\hat{g}_{1}(\zeta)\right| \geqq \frac{1}{2}$ or $\left|\hat{g}_{2}(\zeta)\right| \geqq \frac{1}{2}$ it follows that

$$
\begin{aligned}
& 0 \leqq \log (2 c)-\operatorname{th}(-\eta)+M \log (1+|\xi|+|t \eta|) \\
\text { or } \quad & 0 \leqq \log (2 c)+A|t \eta|-B|\xi|^{\rho}
\end{aligned}
$$

when $P(\xi+i t \eta)=0$ and $\xi, \eta$ and $t>0$ are real. Replacing the constants suitably we have $P(\xi+i t \eta) \neq 0$ if

$$
-\varepsilon|\xi|^{\rho}<t<-C_{1}-C_{2} \log (1+|\xi|+|t|)
$$

when $\eta \in U$ and $\xi$ is large. From the Tarski-Seidenberg theorem we can eliminate the logarithmic term in (4.5) (see the proof of Lemma 2.1 of Appendix in [4] ), which proves (4.1).

Conversely assume that (4.1) is valid. We now define a distribution $E=E_{c_{0}}$ by the identity

$$
E_{\tau_{0}}(x)=(2 \pi)^{-n} \int_{|\xi| \geq \tau_{0}} \frac{e^{i<x, \xi-i t_{1} \vartheta>}}{p\left(\xi-i t_{1} \vartheta\right)} d \xi
$$

with $t_{0}<t_{1}<\varepsilon \tau_{0}^{\rho}$. This means of course the distribution;

$$
\begin{aligned}
C_{0}^{\infty} \ni \check{\varphi} \longmapsto\left\langle E_{\tau_{0}}, \check{\varphi}\right\rangle \\
=(2 \pi)^{-n} \int_{|\xi| \geq \tau_{0}} \frac{\hat{\varphi}\left(\xi-i t_{1} \vartheta\right)}{p\left(\xi-i t_{1} \vartheta\right)} d \xi .
\end{aligned}
$$

The convergence of the integral follows from the Paley-Wiener theorem. Note that $P(D) E_{\tau_{0}}-\delta$ is real analytic and moreover entire holomorphic in $\mathbb{G}^{n}$. Hence, by Ehrenpreis theorem there exists an analytic function $f$ such that $P(D)\left(E_{\tau_{0}}+f\right)=\delta$. What we have to do is thus to show that $\Gamma^{\rho}$ sing supp $E_{\tau_{0}}$ is contained in a $\vartheta$-proper cone. In doing so, let $H=R_{+} U$ and $\Gamma$ be its dual cone, namely

$$
\Gamma=\{x ;\langle x, \xi\rangle \geqq 0 \text { for all } \xi \in H\}
$$

which is a $\vartheta$-proper cone. For a given $x_{0} \notin \Gamma$, let us choose a so small neighborhood $V$ of $x_{0}$ that $V \cap \Gamma=\phi$ and that there is a vector $\eta \in U$ such that with $a>0$

$$
\langle x, \eta\rangle<-a<0, \quad x \in V .
$$


We shall now estimate $\widehat{\varphi E}(\xi), \xi \in \boldsymbol{R}^{n}$, for each $\varphi \in \Gamma_{\mathbf{0}}^{\rho}(V)$. In doing so we may assume that $U$ is connected, by shrinking it if necessary.

Stokes' formula now gives

$$
\begin{aligned}
\widehat{\varphi E}(\xi) & =(2 \pi)^{-n} \int_{\left|\xi^{\prime}\right| \geqq \tau_{0}} \frac{\hat{\varphi}\left(\xi-\xi^{\prime}+i t_{1} \vartheta\right)}{P\left(\xi^{\prime}-i t_{1} \vartheta\right)} d \xi^{\prime} \\
& =(2 \pi)^{-n} \int_{\gamma_{1}} \frac{\hat{\varphi}(\xi-\zeta)}{P(\zeta)} d \zeta \\
& +(2 \pi)^{-n} \int_{\left|\xi^{\prime}\right| \geqq \tau_{0}} \frac{\hat{\varphi}\left(\xi-\xi^{\prime}+i t_{1} \eta\right)}{P\left(\xi^{\prime}-i t_{1} \eta\right)} d \xi^{\prime}
\end{aligned}
$$

where $\gamma_{1}$ is a compact chain in $C^{n}$. If we note that with positive constants $C, B$ and $a$ introduced in (4.6),

$$
\left|\hat{\varphi}\left(\xi-\xi^{\prime}+i s \eta\right)\right| \leqq C \exp \left(-B\left|\xi-\xi^{\prime}\right|^{\rho}-a s\right)
$$

when $\xi, \xi^{\prime}$ and $s$ are real, another application of Stokes' formula gives with $\tau_{1}>0$

$$
\begin{aligned}
\widehat{\varphi E}(\xi)= & (2 \pi)^{-n} \int_{\gamma_{2}} \frac{\hat{\varphi}(\xi-\zeta)}{P(\zeta)} d \zeta \\
& +(2 \pi)^{-n} \int_{\left|\xi^{\prime}\right| \geqq \tau_{1}} \frac{\hat{\varphi}\left(\xi-\xi^{\prime}+i v\left(\xi^{\prime}\right)\right)}{P\left(\xi^{\prime}-i v\left(\xi^{\prime}\right)\right)} d \xi^{\prime}
\end{aligned}
$$

where $v\left(\xi^{\prime}\right)=\varepsilon\left|\xi^{\prime}\right|{ }^{\rho} \eta / 2$ and $\gamma_{2}$ another compact chain. Using (4.7) the first term on the right-hand side of (4.8) can be easily estimated by a constant times $\exp \left(-B \mid \xi^{\rho}\right)$. To do the second term, observe that

$$
\left|P(\xi-i v(\xi))^{-1}\right| \leqq C(1+|\xi|)^{M}
$$

which follows also from the Tarski-Seidenberg theorem. Furthermore we have

$$
\begin{aligned}
\left|\hat{\varphi}\left(\xi-\xi^{\prime}+i v\left(\xi^{\prime}\right)\right)\right| & \leqq C \exp \left(-B\left|\xi-\xi^{\prime}\right|^{\rho}-\frac{a \varepsilon}{2}\left|\xi^{\prime}\right|^{\rho}\right) \\
& \leqq C \exp \left(-C_{1}\left|\xi^{\prime}\right|^{\rho}\right) \exp \left(-C_{2}|\xi|^{\rho}\right)
\end{aligned}
$$

where all the constants are positive and we have used the inequality: $\max$ $\left(\left|\xi-\xi^{\prime}\right|^{\rho},\left|\xi^{\prime}\right|^{\rho}\right) \geqq\left(|\xi|^{\rho} / 4\right)+\left(\left|\xi^{\prime}\right| \rho / 2\right)$. Thus the second term can be estimated also by a constant times $\exp \left(-C_{2}|\xi|^{\rho}\right)$, which means in view of (3.9) and Lemma 3 that $E \in \Gamma^{\rho}$ in $V$, in other words that $\Gamma^{\rho}$ sing supp $E \subset \Gamma$. The 
proof of Theorem 3 is now complete.

When we need not consider the Gevrey class we have in like manner the following theorem, already studied in somewhat different forms by Shirota [9] and Hörmander [5].

Theorem 4. There exists a fundamental solution $E$ of $P(D)$ such that sing supp $E$ is contained in a $\vartheta$-proper cone if and only if there exist positive constants $\varepsilon, \tau_{0}, t_{0}$ and $\rho$ and a neighborhood $U$ of $\vartheta$ such that

$$
p(\xi+i t \eta) \neq 0,-\varepsilon|\xi|^{\rho}<t<-t_{0},|\xi| \geqq \tau_{0}, \text { when } \eta \in U
$$

If $P$ satisfies (4.9) and in addition is not hypoelliptic then we have a fundamental solution $E$ of $P(D)$ whose singular support is contained in a $\vartheta$-proper cone and not equal to the origin. Thus choosing a slab $\Omega=\{x$; $a\left\langle\langle x, \vartheta\rangle\langle b\}\right.$ suitably we have $E \in \mathscr{D}^{\prime}(\Omega)$ such that $P(D) E \in C^{\infty}(\Omega)$ and sing supp $E$ is bounded and actually exists. The requirement, $\eta \in U$, in (4.9) however makes it slightly difficult to obtain examples which satisfy the condition (4.9). But when $P$ is homogeneous, the condition becomes much simpler.

Proposition 1. When $P$ is a homogeneous polynomial, the condition (4.9) is equivalent to the following; there exists a constant $\varepsilon>0$ such that

$$
P(\xi+i t \vartheta) \neq 0 \text { if } 0<t<\varepsilon|\xi|
$$

Proof. We first show the equivalence of (4.9) to the following;

$$
P(\xi+i t \eta) \neq 0 \text { if } 0<t<\varepsilon|\xi| \quad \eta \in U
$$

for $\varepsilon>0$ and a neighborhood $U$ of $\vartheta$. To do so assume that (4.9) is valid for some $\rho$ which may be chosen equal to 1 by the homogeneity of $P$. Since $P(\xi+i t \eta)=\tau^{m} P\left(\tau^{-1} \xi+i \tau^{-1} t \eta\right), P(\xi+i t \eta) \neq 0$ if $-\varepsilon \tau^{-1}|\xi|<\tau^{-1} t<-t_{0}$, which implies $-\varepsilon|\xi|<t<0$ by letting $\tau \rightarrow 0$. Since that (4.11) implies (4.9) is trivial, our claim is now verified. In order to prove that (4.10) means (4.11) we introduce the localization $P_{\xi}$ of $P$ at $\xi \in \boldsymbol{R}^{n} \backslash 0$, following AtiyahBott-Gårding [2] and Andersson [1], which is defined as the coefficient of the lowest order term in $\tau$ when we develop $P(\xi+\tau \zeta)$ around $\xi \in \mathbb{R}^{n} \backslash 0$; 


$$
P(\xi+\tau \zeta)=\tau^{p} P_{\xi}(\zeta)+\text { terms of higher order in } \tau
$$

where $p$ is called the multiplicity of $P$ at $\xi$. If $P$ satisfies (4.10) and $P_{\xi_{0}}$ $(\xi+s \vartheta), \xi \in \boldsymbol{R}^{n}$, has a root $s_{0}$ with $\operatorname{Im} s_{0} \neq 0$, in view of the identity $\tau^{-p} P\left(\xi_{0}+\tau(\xi+s \vartheta)\right)=P_{\xi_{0}}(\xi+s \vartheta)+0(|\tau|), P\left(\xi_{0}+\tau \xi+\tau s \vartheta\right)$ also has a root $s_{1}$ near $s_{0}$, in particular with $\operatorname{Im} s_{1} \neq 0$, for sufficiently small $\tau>0$, which contradicts the condition (4.10). It thus follows that $P_{\xi_{0}}(\xi+s \vartheta) \neq 0$ when $\xi \in \boldsymbol{R}^{n}$ and $\operatorname{Im} s \neq 0$, which means that $P_{\xi_{0}}$ is hyperbolic with respect to $\vartheta$. Now that $P(\xi+\cdot)$ is locally hyperbolic following the terminology of Gårding [3], we have (4.11) from Main Lemma in [3]. However, for the convenience of the reader, we shall here copy the proof from [3]. We consider the function

$$
f(s, t, u, \eta)=\tau^{-p} P\left(\xi_{0}+\tau(\xi+s \vartheta+t \eta)\right)
$$

where $\left|\xi_{0}\right|=1, u=(\tau, \xi), p$ the multiplicity of $P$ at $\xi_{0}$ and $\eta$ belongs to a compact convex neighborhood $K$ of $\vartheta$ where $P_{\xi_{0}}$ does not vanish. Since $f(0, t, 0, \eta)=t^{p} P_{\xi_{0}}(\eta)$, we have when $s, t, u$ are small

$$
f(s, t, u, \eta)=P_{\xi_{0}}(\eta) \prod_{1}^{p}\left(t+\lambda_{k}(s, u, \eta)\right) F(s, t, u, \eta)
$$

where $\lambda_{1}, \ldots, \lambda_{p}, F$ are continuous and $F(0,0,0, \eta)=1$ Because the hyperbolicity of $P_{\xi_{0}}$ implies that $P_{\xi_{0}}(s \vartheta+t \eta) \neq 0$ when $0<\operatorname{Im}(s+t)$ and because of (4.10), we have $\operatorname{Im} \lambda_{k}(s, 0, \eta)>0$ and $\operatorname{Im} \lambda_{k}(s, u, \eta) \neq 0$ when $\operatorname{Im} s>0$ and real $u, s, t$ are small. Thus we have $\operatorname{Im} \lambda_{k}(s, u, \eta) \geqq 0$ when $\operatorname{Im} s \geqq 0$ and real $u, s, t$ are small. Putting $s=0$ in (4.12), we obtain with $\varepsilon_{0}>0$,

$$
P\left(\xi_{0}+\tau \xi+t \eta\right) \neq 0, \quad 0<\operatorname{Im} t<\varepsilon_{0},
$$

when $\eta \in K$ and real $\tau,|t|, \xi$ are small. Moving $\xi_{0}$ with $\left|\xi_{0}\right|=1$, we have (4.11) by the compactness of the sphere.

Examples. All the polynomials written in products of hypoelliptic and hyperbolic polynomials satisfy the condition (4.9). $P\left(\xi_{1}, \xi_{2}, \xi_{3}\right)$ $=\xi_{1}^{4}-\xi_{2}^{4}-\xi_{3}^{4}$ which is irreducible and neither hyperbolic nor hypoelliptic satisfies the condition (4.9) when $\vartheta=(1,0, \ldots, 0)$ in view of Proposition 1. 


\section{References}

[1] Andersson, K. G., Propagation of analyticity of solutions of partial differential equations with constant coefficients, Ark. Mat. 8 (27) (1971) 277-302.

[2] Atiyah, M. F., Bott, R. and Gårding, L., Lacunas for hyperbolic differential operators with constant coefficients I, Acta. Math. 124 (1970) 109-189.

[3] Gårding, L., Local hyperbolicity, Israel J. Math. 13 (1972) 65-81.

[4] Hörmander, L., Linear partial differential operators. Springer, 1963.

[5] Hörmander, L., On the singularities of solutions of partial differential equations, Comm. Pure. Appl. Math. 23 (1970), 329-358.

[6] John, F., Non-admissible data for differential equations with constant coefficients. Comm. Pure. Appl. Math. 10 (1957) 391-398.

[7] Kashiwara, M., On C-hyperbolic differential operator with constant coefficients, SurikaisekiKenkyusho Kokyuroku 145, R.I.M.S., Kyoto Univ., (1972) 168-171 (Japanese).

[8] Matsuura, S., On the propagation of support of solutions to general systems of partial differential equations, Int. Symp. on Mathematical Problems in Theoretical Phycics, Kyoto, Japan, (1975), to appear in Lecture Notes in Physics.

[9] Shirota, T., On the propagation of regularity of solutions of partial differential equations with constant coefficients, Proc. Japan Acad., 38, (1962), 587-590. 
